\title{
Proton transport through self-generated waves in impulsive flares
}

\author{
R. Vainio* and L. Kocharov
}

Space Research Laboratory, Department of Physics, University of Turku, 20014 Turku, Finland

Received 28 March 2001 / Accepted 28 May 2001

\begin{abstract}
Energetic proton transport through self-generated Alfvén waves in impulsive ( $\gamma$-ray) flares is studied using the method of Monte Carlo simulations. Protons are traced inside a flux tube after they are released from a point source located inside the loop until they hit the boundary of the 1-D simulation box and escape. As they stream from the source towards the boundaries, the particles generate Alfvén waves through the streaming instability. We consider both open and closed field lines. In the closed field line case, the escaping particles precipitate and produce observable secondary emissions; for the open field line, particles precipitate only from one end of the field line, and escape freely to the interplanetary medium from the other end. For a sufficiently large number of accelerated protons per unit area, $\gg n_{0} V_{\mathrm{A}} / \Omega_{\mathrm{p}}$ where $n_{0}$ is the plasma density, $V_{\mathrm{A}}$ the Alfvén speed, and $\Omega_{\mathrm{p}}$ the proton gyro-frequency, the particle flux from the source produces a turbulent trap that expands at Alfvén speed in both directions from the source. The resulting $\gamma$-ray emission from the loop legs consists of a precursor, related to the quick propagation of particles when the trap has not formed yet, and of a delayed brightening in the loop leg closer to the source, related to the opening of the turbulent trap as the self-generated waves reach the solar surface. For impulsive injections lasting $\ll L /\left(2 V_{\mathrm{A}}\right)$, where $L$ is the loop length, the second emission may be suppressed by adiabatic deceleration in the expanding turbulent trap. For open field lines, our model is capable of producing the small ratio of the numbers of interplanetary-to-interacting protons typically observed in impulsive flares, if the proton source is located close to the Sun.
\end{abstract}

Key words. diffusion - instabilities - Sun: flares - Sun: particle emission - Sun: X-rays, gamma rays - turbulence

\section{Introduction}

Acceleration of protons up to several tens of $\mathrm{MeVs}$ and beyond may take place at different phases of solar eruptions, from the flare pulse in the low corona through the passage of the interplanetary shock beyond the Earths orbit. Some protons interact at the Sun, producing secondary $\gamma$-rays and neutrons, others escape into the interplanetary medium to produce a solar energetic particle (SEP) event at $1 \mathrm{AU}$. The current two-class paradigm of solar particle events suggests that impulsive SEP events originate in impulsive solar flares and gradual events are linked to the coronal/interplanetary shocks (Cane et al. 1986; Cliver 1996; Reames 1999 and references therein). Impulsive flares may produce ${ }^{3} \mathrm{He}$-rich SEP events, which typically are not strong in terms of the total number of accelerated protons. Some impulsive flares, however, can produce a large number of interacting protons and can be observed in the $\gamma$-ray band. In such events the ratio of the numbers of interplanetary-to-interacting protons is typically small $(\$ 0.1)$, whereas gradual $\gamma$-ray flares show larger $(\gtrsim 1)$ values of this ratio (Kocharov \& Kovaltsov 1986; Hua \& Lingenfelter 1987; Ramaty et al. 1993).

Send offprint requests to: R. Vainio, e-mail: ravainio@utu.fi

* Supported by the Academy of Finland (project 46331).
Intensive production of accelerated protons implies the potential importance of self-generated waves, i.e., waves generated by the flux of protons leaking from the accelerator (see, e.g., $\mathrm{Ng}$ et al. 1999 in application to gradual SEP events). In application to impulsive $\gamma$-ray flares, the role of self-generated waves was emphasized by Bespalov et al. $(1987,1991)$. The transport conditions of protons in flaring loops determine the time scale for their precipitation. The conditions are, therefore, crucial for the flare scenario. Strong interaction with hydromagnetic waves is known to make the trapping of protons inside the loop efficient. Bespalov et al. $(1987,1991)$ studied the proton transport under the assumption that the protons stream away from a source located at the top of the loop, and generate Alfvén waves. They showed that for a sufficiently strong source, the waves would become so intense that the particles would essentially be locked on the waves and convect with them at Alfvén speed towards the footpoints of the loop. Electrons, being coupled to whistler and ion-cyclotron waves, would travel much faster than protons, having a bulk speed of $\$ 9 V_{\mathrm{A}}$ (e.g., Vainio 2000). This would lead, consistent with observations of impulsive flares, to a delay in the $\gamma$-ray emission relative to the X-ray emission, if electrons and protons were accelerated simultaneously near the top of the flaring loop (Bespalov et al. 1987). Since the bulk speed of electrons 
depends on energy, however, the ultra-relativistic electrons responsible for the $\gamma$-ray continuum behave very similarly to protons. Note that the delay of the peak time of the $\gamma$-ray emission relative to the X-ray peak could also be interpreted in terms of particle transport in flaring loops with weak external turbulence and large variation of the cross-sectional area of the flux tube (Hulot et al. 1989, 1992).

One aspect of the turbulent trapping model missing from the original work of Bespalov et al. $(1987,1991)$ is that it could lead to interesting effects, if the flux tube is asymmetric with respect to the source position, and if transient processes are incorporated in the model. Scattering off self-generated waves at open magnetic field lines may also affect the interplanetary-to-interacting proton ratio. Vainio et al. (2000) obtained reasonable ratios for gradual flares in a model of coronal shock acceleration by arranging the turbulence around the shock in a stationary manner to mimic self-generated waves. In the case of impulsive flares, the role of self-generated waves in forming the interplanetary-to-interacting proton ratio has not yet been studied. Applying the asymmetric, strongly turbulent transport model to an open flux tube with the solar surface on one end, and a free-escape boundary, i.e., the interplanetary medium, at the other end, we could anticipate a small value of the interplanetary-to-interacting proton ratio, if the source is close to the Sun.

The purpose of this paper is to study and extend the transport model of Bespalov et al. (1987, 1991), using a time-dependent numerical method. To simplify the model at this stage, we perform the study inside a single magnetic flux tube with constant values of the magnitude of the magnetic field and plasma density, and zero bulk speed of the thermal plasma. The study includes as separate cases a symmetric loop (Case A), an asymmetric loop (Case B), and an open field line (Case C). The potentially important effects of non-constant plasma parameters (see, e.g., Kocharov et al. 1999) is left for the future. We note here, however, that interpretation of magnetogram data (McClymont \& Mikic 1994) suggest that coronal loops showing almost constant cross section are possible due to twists in the magnetic flux tubes. Thus, the constant-field approximation should not be too far from reality to be applied in this study.

\section{The model}

Our model consist of a (possibly curved) magnetic field with a constant magnitude along the line of force considered. Either one (Case C) or both (Cases A and B) ends of the flux tube are tied to the solar surface, where we assume that the density of the plasma is so large that all particles reaching this region interact, producing secondary emissions. No mirroring of particles at the footpoints of the flux tube is taken into account. Trapping of protons by mirroring at footpoints leads to the escape time of $2 \rho_{0}(L / v)$ (e.g., Hulot et al. 1989), where $\rho_{0}$ is the footpoint mirror ratio. If this time is small relative to the trapping time by self-generated waves, $L /\left(2 V_{\mathrm{A}}\right)$ (Bespalov et al. 1987, 1991), one may regard neglecting the footpoint mirroring reasonable (cf. Kocharov et al. 1999). Thus, this approximation is justified at $\rho_{0} \ll v /\left(4 V_{\mathrm{A}}\right)$, but for very large values of $\rho_{0} \gtrsim v /\left(4 V_{\mathrm{A}}\right)$, trapping by footpoint mirroring should become important. The basic scale length of the (1-D) system is denoted by $L$. In the case of a closed magnetic field, $L$ is the length of the loop and in the case of an open field, $L$ is the height at which the particles are assumed to freely escape into the interplanetary medium.

The plasma parameters are fixed to representative values for flaring loops (Bespalov et al. 1987): Alfvén speed $V_{\mathrm{A}}=10^{8} \mathrm{~cm} \mathrm{~s}^{-1}$, plasma electron (and proton) density $n_{0}=10^{11} \mathrm{~cm}^{-3}$, and the length of the loop $L=10^{9} \mathrm{~cm}$. In Case $\mathrm{C}$, we choose the length of the trapping region as $L=5 \times 10^{9} \mathrm{~cm}$ being less than or of the order of the scale height of the magnetic field in the lower corona. The magnetic field in such a flux tube (with electron-proton composition) is $B \approx 145$ Gauss, and the ion skin length, $V_{\mathrm{A}} / \Omega_{\mathrm{p}} \approx 72 \mathrm{~cm}$, where $\Omega_{\mathrm{p}} \approx 1.4 \times 10^{6} \mathrm{~s}^{-1}$ is the proton gyro-frequency. The plasma temperature is taken to be $T=10^{7} \mathrm{~K}$ for both electrons and protons. This gives a value of plasma beta of $\beta=16 \pi n_{0} k_{\mathrm{B}} T / B^{2} \approx 0.33$ for the thermal component.

The Alfvén waves responsible for the collisionless scattering of the accelerated protons are assumed to propagate along the mean magnetic field. We, therefore, assume that the flux tube acts as a wave guide in case it is curved. This is valid, if the considered flux tube has a higher plasma density than its surroundings (Mazur \& Stepanov 1984). For simplicity, no absorption of the waves by the thermal plasma nor any wave-wave interactions are thought to occur. Sunward-propagating waves are absorbed and antisunward propagating waves are emitted at the footpoints of the flux tube. The emitted wave flux at the footpoints has to be, of course, given as a boundary condition.

We consider energetic protons of momentum $p=p_{0}$ emitted from a point source located inside the flux tube at $x=x_{0}$. Protons are followed under the guiding-center approximation along the magnetic field as they undergo wave-particle interactions with Alfvén waves of wavenumber that is assumed to be fixed and given by $k=m \Omega_{\mathrm{p}} / p_{0}$. This approximation to the full resonance condition, $k=$ $m \Omega_{\mathrm{p}} / p \mu$ ( $p \mu$ is the parallel momentum in the wave frame), allows us to follow a single wavenumber instead of the full spectrum of them, and makes the simulations much simpler and faster. Particles hitting the ends of the flux tube are assumed to be absorbed (by stopping at the solar surface or by escape to the interplanetary medium). The wave-particle interactions are modeled as pitch-angle scattering that is elastic in each wave frame and occurs at the scattering rates (Skilling 1975)

$\nu_{ \pm}(x, p, t)=\frac{\pi}{4} \Omega \frac{U_{ \pm}(x, t)}{U_{B}}$

where $U_{B}=B^{2} / 8 \pi, \Omega=\Omega_{\mathrm{p}} / \gamma$ is the relativistic gyro-frequency of the proton, and $U_{ \pm}(x, t)$ is the total (kinetic+magnetic) energy density (per logarithmic 
bandwidth in wavenumber) of waves propagating parallel $(+)$ or anti-parallel $(-)$ to the field line. The scatterings lead to pitch-angle diffusion with isotropic diffusion coefficients $D_{\mu \mu}^{ \pm}=\frac{1}{2}\left(1-\mu^{2}\right) \nu_{ \pm}$.

Energetic protons interact with the waves selfconsistently in the sense of conserving the total energy (as measured in the plasma frame) of waves and particles at a microscopic level. Let $p_{ \pm}$and $\mu_{ \pm}$denote the momentum and pitch-angle cosine as measured in the wave frame indicated by the subscript. A particle scattering in pitch-angle cosine by an amount of $\Delta \mu_{ \pm}$(in the wave frame) suffers an energy loss of $-\Delta E=\mp V_{\mathrm{A}} p_{ \pm} \Delta \mu_{ \pm}$in the plasma frame. We use isotropic scattering, so $\left\langle\Delta \mu_{ \pm}\right\rangle / \Delta t=\partial D_{\mu \mu} / \partial \mu=$ $-\mu_{ \pm} \nu_{ \pm}$. This leads to a growth of the waves at the rate

$$
\begin{aligned}
\Gamma^{ \pm} & = \pm \frac{1}{U_{ \pm}} \int \mathrm{d}^{3} p_{ \pm} \nu_{ \pm} V_{\mathrm{A}} p_{ \pm} \mu_{ \pm} f_{ \pm} \\
& = \pm \Omega_{\mathrm{p}} \frac{\pi S_{ \pm}}{2 n_{0} V_{\mathrm{A}}}
\end{aligned}
$$

where $f_{ \pm}\left(\mu_{ \pm}, p_{ \pm}, x, t\right)$ is the distribution function of the accelerated particles and $S_{ \pm}=\int \mathrm{d}^{3} p_{ \pm} v_{ \pm} \mu_{ \pm} f_{ \pm}$gives their flux. A more detailed description of the numerical method is given in the Appendix.

\section{Results}

\subsection{Case A: Symmetric loop}

In the first set of simulations, we study a loop which is spatially symmetric about the point source of energetic protons. We inject $p_{0}=0.25 \mathrm{mc}$ (corresponding to an energy of about $30 \mathrm{MeV}$ ) protons isotropically at a rate $Q=\epsilon n_{0} V_{\mathrm{A}}=\epsilon \times 10^{19} \mathrm{~cm}^{-2} \mathrm{~s}^{-1}$, where $\epsilon=2 \times 10^{-5}$ is chosen to give a large flux resulting in a rapid wave growth, but still keeping the energetic-proton pressure at least an order of magnitude below the thermal proton pressure even if all particles are trapped by Alfvén waves inside $|x|<V_{\mathrm{A}} t$. We vary the total amount of injected particles by varying the duration of the injection between $\Delta t=1 / 15 \mathrm{~s}$ and $10 / 15 \mathrm{~s}$, resulting in a total number of injected particles between $Q \Delta t=1.33 \times 10^{13} \mathrm{~cm}^{-2}$ and $1.33 \times 10^{14} \mathrm{~cm}^{-2}$. The background wave flux emitted from the footpoints is fixed by assuming that the wave mode emitted from each footpoint has an energy density of $2 U_{ \pm}(\mp L / 2, t)=2 U_{0}=10^{-4} \times U_{B}$. We assume that this emission of waves is steady; thus the initial condition for both wave modes is also $U_{ \pm}(x, 0)=U_{0}$. The adopted value for $2 U_{0}$ corresponds to an rms velocity amplitude of $10 \mathrm{~km} \mathrm{~s}^{-1}$ per logarithmic bandwidth at $f=V_{\mathrm{A}} k / 2 \pi \sim 3 \mathrm{kHz}$. Assuming that this velocity amplitude holds for all frequencies from $f_{0}=V_{\mathrm{A}} / L=0.1 \mathrm{~Hz}$ up to $f_{\mathrm{p}}=\Omega_{\mathrm{p}} / 2 \pi \approx 220 \mathrm{kHz}$, the total rms amplitude is $38 \mathrm{~km} \mathrm{~s}^{-1}$, which agrees very well with the typical observed non-thermal velocity in a quiescent active region of $45 \mathrm{~km} \mathrm{~s}^{-1}$ (Antonucci \& Dodero 1995). The non-thermal velocities in flaring plasmas are larger, consistent with wave growth. We do not consider any emission of waves from the acceleration site; we assume that the waves are absorbed by the particles during the acceleration. However, some external forcing of the Alfvénic turbulence is modeled by keeping the wave-energy densities above a minimum level of $U_{ \pm} \geq U_{\min }=5 \times 10^{-7} \times U_{B}$ everywhere inside the flux tube.

The results of the simulations are presented in Fig. 1 in form of flux of protons precipitating at the footpoints of the loop. One can see a peak in the precipitating flux at $t \approx 5 \mathrm{~s}$ corresponding to the travel time of Alfvén waves from the center of the loop to the footpoints, as predicted by the theory of Bespalov et al. $(1987,1991)$. What is not predicted by the steady-state theory is the rather intense precursor peak immediately after the particle release, that corresponds to the first phase, when the waves have not yet grown enough to suppress the diffusive particle transport. The number of particles in the precursor seems to be independent of the number of injected particles, if this number exceeds a threshold level. This can be understood as follows. We consider an initial turbulence level, where the diffusion length, $\kappa / V_{\mathrm{A}}$ (where $\kappa=\frac{1}{3} v^{2} / \nu$ is the spatial diffusion coefficient), is larger than the distance from the source to the footpoints. Until the wave-energy density has grown to a level, denoted by $U^{*}$, that suppresses diffusive transport, the particles propagate quickly (relative to the time scale of wave transport) towards the footpoints adjusting the value of particle flux to $\left|S_{ \pm}\right| \approx \frac{1}{2} Q$ (directed away from the source). The wave-energy density obeys

$$
\begin{aligned}
\ln \frac{U_{ \pm}(x, t)}{U_{0}} & =\int_{0}^{t} \mathrm{~d} t^{\prime} \Gamma_{ \pm}\left(x \mp V_{\mathrm{A}} t^{\prime}, t^{\prime}\right) \\
& = \pm \Omega_{\mathrm{p}} \frac{\pi}{2 n_{0} V_{\mathrm{A}}} \int_{0}^{t} \mathrm{~d} t^{\prime} S_{ \pm}\left(x \mp V_{\mathrm{A}} t^{\prime}, t^{\prime}\right) \\
& \approx \pm \Omega_{\mathrm{p}} \frac{\pi}{4 n_{0} V_{\mathrm{A}}} Q t
\end{aligned}
$$

where the approximation on the last line is valid until the time $t^{*}$ when the waves have reached the level $U^{*}$. This can be turned around to give the number of precursor particles per unit area, $\mathrm{d} N_{\text {prec }} / \mathrm{d} A=Q t^{*}$, as

$$
\frac{\mathrm{d} N_{\mathrm{prec}}}{\mathrm{d} A} \sim \frac{4}{\pi} \frac{n_{0} V_{\mathrm{A}}}{\Omega_{\mathrm{p}}} \ln \frac{U^{*}}{U_{0}}
$$

It is not necessary to know the value of $U^{*}$ exactly since it appears in the logarithm, and the numerical factor can be determined from simulations. However, a lower-limit estimate is given by equating the diffusion length to the distance from the source to the footpoint, i.e.,

$$
\frac{4}{3 \pi} \frac{U_{B}}{U^{*}} \frac{v^{2}}{V_{\mathrm{A}} \Omega} \sim \frac{L}{2}
$$

giving

$U^{*} \sim \frac{8}{3 \pi} \frac{\gamma v^{2}}{V_{\mathrm{A}}^{2}} \frac{V_{\mathrm{A}}}{L \Omega_{\mathrm{p}}} U_{B}=\frac{4}{3 \pi} \frac{V_{\mathrm{A}}}{L \Omega_{\mathrm{p}}} p v n_{0}$

Inserting the numerical values gives $U^{*} / U_{0} \sim 7$ and $\mathrm{d} N_{\text {prec }} / \mathrm{d} A \sim 10^{13} \mathrm{~cm}^{-2}$, which is seen to agree well with the number of precursor particles in the simulation. 


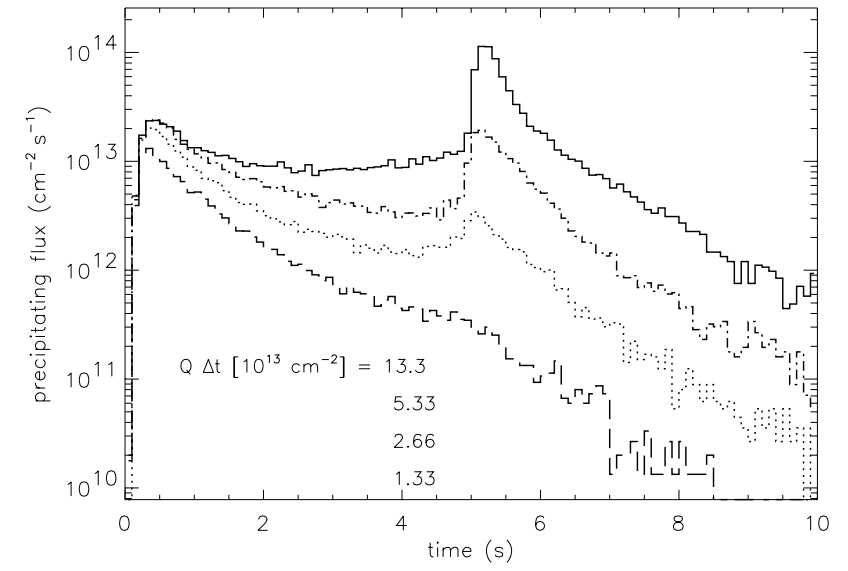

Fig. 1. Flux of precipitating particles for four values of the total number of injected protons. The rate of injection is kept constant, $Q=2 \times 10^{-14} \mathrm{~cm}^{-2} \mathrm{~s}^{-1}$, and its duration, $\Delta t$, is varied, as indicated with their product in the legend.

Let us estimate the spectrum of the promptlyprecipitating protons. If a spectrum of particles is emitted, and if energy changes during the transport are neglected, we should identify the number of precursor protons with $Q t^{*}=p \mathrm{~d} N_{\text {prec }} /(\mathrm{d} A \mathrm{~d} p)$ in the spirit of the resonance condition $k \propto p^{-1}$. For a wave spectrum $U_{0} \propto p^{q-1}$ with $q<3, p \mathrm{~d} N_{\text {prec }} /(\mathrm{d} A \mathrm{~d} p)$ increases with (non-relativistic) energy, although logarithmically $\left(\propto \ln v p^{2-q}\right)$. Typical turbulence models give $1<q<2$. Up to the energy where the total number of injected particles (per logarithmic momentum interval), $p \mathrm{~d} N /(\mathrm{d} A \mathrm{~d} p)$, equals the calculated value for the precursor, Eqs. (4-6), the energy spectrum of the first peak is $\mathrm{d} N_{\text {prec }} /(\mathrm{d} A \mathrm{~d} E) \propto\left(\ln v p^{2-q}\right) /(v p)$. At larger energies, only the first peak is observed with a spectrum identical to that of the source.

Using the assumption of a steady state, Bespalov et al. (1991) deduced that the rate of particle injection determines whether particle transport is governed by diffusion or convection with the waves. That is correct in the steadystate regime of continual injection. In the case of short injection, however, the total number of injected particles, $Q \Delta t$, is the only controlling factor in the relative importance of the prompt and delayed components of the precipitating flux. To confirm this, we ran a simulations of using injection rates of $Q=2 \times 10^{-6} n_{0} V_{\mathrm{A}}, 2 \times 10^{-5} n_{0} V_{\mathrm{A}}$, and $1 \times 10^{-4} n_{0} V_{\mathrm{A}}$ and with $Q \Delta t=6.67 \times 10^{13} \mathrm{~cm}^{-2}$ in all cases (Fig. 2). The resulting flux of precipitating particles looks nearly identical for the shortest injections $(\Delta t=1 / 15 \mathrm{~s}$ and $1 / 3 \mathrm{~s})$ since the diffusive time scale is large enough to regard both of these injections as impulsive. The prolonged injection $(\Delta t=10 / 3 \mathrm{~s})$, however, suppresses the height of the precursor while keeping the total number of precursor particles very similar to the impulsive ones, which is in accord with diffusive transport. Further, increasing the loop length or the level of ambient turbulence inside the loop clearly suppresses the importance of the precursor predicted by the estimation and confirmed by simulations. We, thus, regard the Eqs. (4-6),

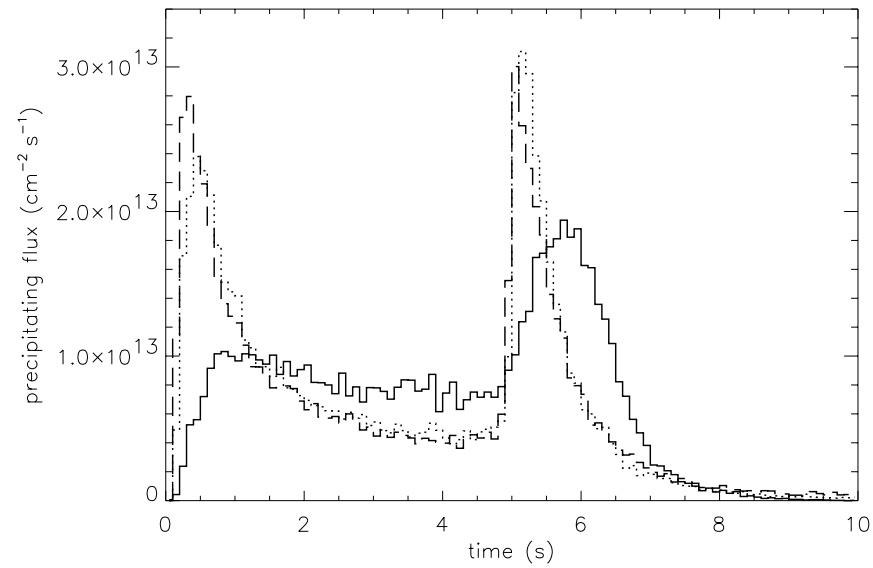

Fig. 2. Precipitating flux for a constant number of injected particles, $Q \Delta t=6.67 \times 10^{13} \mathrm{~cm}^{-2}$, and a variable duration of the injection, $\Delta t=1 / 15 \mathrm{~s}$ (dashed curve), $1 / 3 \mathrm{~s}$ (dotted curve), and $10 / 3 \mathrm{~s}$ (solid curve).

up to a numerical factor of the order unity, as a valid scaling law for arranging observations of precipitating proton fluences.

\subsection{Case B: Asymmetric loop}

As became evident from the simulations of Case A, the majority of the particles in a powerful impulsive flare precipitate once the turbulent walls have reached the footpoints of the flux tube, at $t=L / 2 V_{\mathrm{A}}$. It is of interest to investigate what happens if the particles are not released in the central point of the flux tube; if the trap reaches one end first, we should see a peak in the precipitating flux at this footpoint, but if the precipitation is rapid, the particle reservoir might empty before the trap opens from the other end. We have simulated this by varying the position of the source as $x_{0}=0.05 L, 0.1 L$, and $0.25 L$ (from the center of the loop). The number of injected particles was $6.67 \times 10^{13} \mathrm{~cm}^{-2}$, and other parameters were chosen as in Case A. The results are plotted in Fig. 3. The flux towards the footpoint closer to the source resembles the symmetric case with a prompt and a delayed peak. The flux at the other footpoint, however, behaves differently. Even a slightly asymmetric position of the source can lead to a huge difference in the precipitating flux for the delayed component; in practice, we can expect a delayed brightening in $\gamma$-rays at only one of the footpoints based on our simulations. However, the observed asymmetry in the precursor brightening provides a measure of the asymmetry of the source position, if the ambient turbulence level is high enough to yield a diffusive delay in the particle flux reaching the footpoints at different distances from the source.

Without a possibility to distinguish between the emission from different footpoints, the observations in the asymmetric case are very similar to the symmetric case, and the general conclusions drawn from Case A - two-component structure, importance of the 


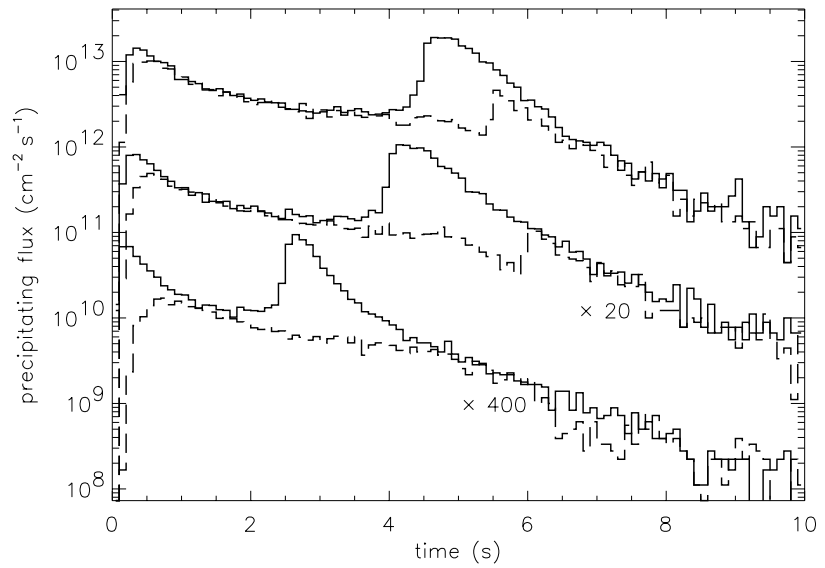

Fig. 3. The flux of precipitating protons in a loop with an asymmetric source position at $x_{0}=0.05 \mathrm{~L}, 0.1 \mathrm{~L}$, and $0.25 \mathrm{~L}$ (top to bottom). The curves should be multiplied by the indicated constants. Solid curves correspond to the footpoint closer to and dashed curves to the footpoint farther from the source. The total number of injected $30-\mathrm{MeV}$ protons is $6.67 \times 10^{13} \mathrm{~cm}^{-2}$.

total number of injected particles - are valid also for the asymmetric case.

\subsection{Case C: Open flux tube}

As a last study, we performed simulations in a flux tube that is connected to the solar surface from one footpoint at $x=0$, only. The other end at $x=L=5 \times 10^{9} \mathrm{~cm}$ is assumed to leak the incident particles directly to the interplanetary medium, modeling a flux tube rapidly expanding above $x=L$; a large and rapid expansion would probably eject most of the particles reaching this distance, since the particle bulk speed is directed outwards, and particles diffusing backward relative to the flow at $x \gtrsim L$ see the boundary as a strong magnetic mirror. In a more gently opening geometry, the effects of the diverging field should be taken into account explicitly in the simulation. The source is positioned at $x=0.1 L$, and the total number of injected particles is $1.33 \times 10^{14} \mathrm{~cm}^{-2}$. This case differs from Case B simulations in that we assume that the background Alfvén waves have a large cross helicity: the outward propagating waves have $U_{+}(x=0, t)=U_{+}(x, t=$ $0)=U_{0}=5 \times 10^{-5} \times U_{B}$ as in Cases $\mathrm{A}$ and $\mathrm{B}$, but the inward propagating waves have only the minimum-level intensity, $U_{\min }=5 \times 10^{-7} \times U_{B}$. Other parameters were taken as in Cases A and B.

The results of the open-field simulation are shown in Fig. 4. The precipitating particle flux again shows a double peaked structure, with a precursor well described by Eqs. (4-6). The escaping flux, however, rises more slowly to a stationary level that is destroyed by the boundary effects, when the turbulent trap hits the free-escape boundary, opening it also from this end. Towards the end of the event, the escaping flux becomes dominant over the precipitating flux. The number of precipitating particles is, however, an order of magnitude larger than the number

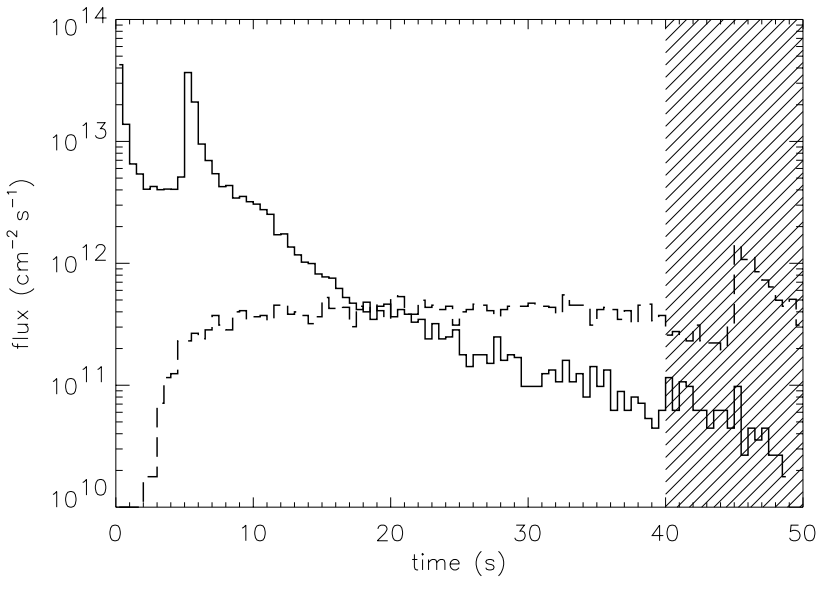

Fig. 4. The flux of particles precipitating (solid curve) and escaping into the interplanetary medium (dashed curve) in an open magnetic flux tube. The hatched area is disturbed by a somewhat oversimplified outer boundary condition.

of escaping particles, which shows that energetic particles accelerated in impulsive flares can be effectively trapped near the Sun even on open field lines. This demonstrates that the small ratio of interplanetary-to-interacting protons observed in impulsive flares do not necessarily result from closed field line topology.

\section{Discussion and conclusions}

We have modeled the transport of energetic protons in impulsive flares through self-generated waves using the Monte Carlo method. Our model uses a scattering law that is proportional to the energy densities of single- $k$ Alfvén waves propagating in both directions along the mean field. The energy lost (gained) by the particles in the scatterings is given to (taken from) the waves. The full quasi-linear resonance condition would involve a spectrum of waves even in case of mono-energetic particle distribution. The artificially sharpened resonance condition $\alpha_{0} k p=m \Omega_{\mathrm{p}}$, where $\alpha_{0}$ is a numerical constant of the order unity, is, however, a commonly used approximation in diffusive particle transport and acceleration theories involving self-generated waves (e.g., Skilling 1975; Bell 1978; Lee 1983). It introduces an inaccuracy to the scattering rates, but for a power-law spectrum of particles it can be tuned by choosing $\alpha_{0}$ properly to yield correct growth rates for waves (Skilling 1975). Our simulations make use of a still simpler form, $k \propto p_{0}^{-1}$, which is a reasonable choice, if the particles approximately conserve their energies during the propagation. Note that this assumption is made implicitly in the analytical calculations of Bespalov et al. (1987, 1991). Because the turbulent trap is expanding along the magnetic field at a constant rate, however, there exists adiabatic deceleration of the trapped particles at a rate $\dot{p} / p=-1 /(3 t)$. This estimate is the lower limit, since it assumes that the particles spend a negligible fraction of time scattering off the turbulent walls of the trap and that stochastic acceleration inside the trap can be 




Fig. 5. Average momentum of precipitating particles in Case A as a function of time for $Q=2 \times 10^{14} \mathrm{~cm}^{-2} \mathrm{~s}^{-1}$. The curves are as in Fig. 1.

neglected. However, if the particle is trapped at $t=t_{0}$, it has an average final momentum of $\langle p\rangle=p_{0}\left(2 V_{\mathrm{A}} t_{0} / L\right)^{1 / 3}$, when the trap opens (symmetric case). Thus, significant deceleration could be expected, if the characteristic trapping time $t_{0}$ is small. The simulations reveal that precipitating particles have average momenta $\ln \langle p\rangle / p_{0}>-1$ (see Fig. 5), so the approximative resonance condition seems acceptable for scattering estimates. Note, however, that the change in energy during trapping can be substantial in the context of $\gamma$-ray production, up to a factor of $\sim 5$ in the non-relativistic case. For a typical integral particle spectrum, $N(>E) \propto E^{-2}$, this means a factor of $\sim 25$ fewer particles capable of $\gamma$-ray production, and may suppress the delayed peak of emission completely. Note, however, that the problem of adiabatic deceleration is most severe in the case of strong, impulsive injections, as indicated by Fig. 6, where the average momentum is plotted for a constant number of injected particles varying the duration of the injection; for prolonged injections with $\Delta t \gtrsim L /\left(2 V_{\mathrm{A}}\right)$, the particles conserve their energies more effectively, because most of them are injected in a trap that has a large initial dimension.

Another simplification in our model is the assumption of constant magnetic field and plasma density inside the loop. A constant magnetic field is often seen as near-uniform cross sections of the flare loops (McClymont \& Mikic 1994). Non-constant plasma parameters, however, affect wave propagation, particle transport, and wave growth in several ways: (i) wave-frequency conservation changes the wavenumber of a wave propagating at a spatially varying phase speed; (ii) resonant wavenumber changes with position in a non-constant magnetic field; (iii) particles suffer mirroring and adiabatic energy changes in a non-constant magnetic field. Because of all these effects, the wave growth is also affected. Our next step in developing the code is to replace the single wave fields and mono-energetic injections with with wavenumber and particle-energy spectra to estimate the effects of non-constant plasma parameters on wave growth. Once

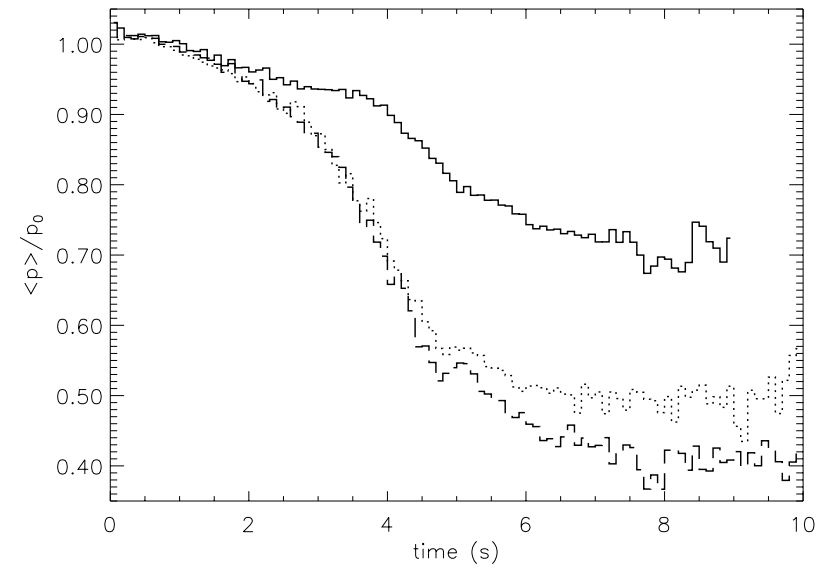

Fig. 6. Average momentum of precipitating particles in Case A as a function of time for $Q \Delta t=6.67 \times 10^{13} \mathrm{~cm}^{-2}$ and a variable duration of the injection, $\Delta t=1 / 15 \mathrm{~s}$ (dashed curve), $1 / 3 \mathrm{~s}$ (dotted curve), and 10/3 s (solid curve).

this is done, the resonance conditions can also be modeled more realistically.

Wave damping by plasma ions should be much less important than the growth or decay due to the energetic particles, because we are considering non-compressive Alfvén waves having only gyro-resonant interactions. We note, however, that if one drops the assumption of a waveguiding loop, even initially parallel-propagating righthanded (left-handed) Alfvén waves convert to oblique fast-magnetosonic (Alfvén) waves and may suffer Landau damping (Wentzel 1976). From other processes, at least wave-wave interactions, as described by weak-turbulence theory, between Alfvén waves $\left(A_{\llcorner}^{ \pm} \rightleftharpoons A_{\perp}+A_{L}^{\prime \pm}\right)$ and between Alfvén waves and sound waves $\left(A_{\|}^{ \pm} \rightleftharpoons S^{ \pm}+A_{\|}^{\mp}\right)$ have potential importance (see, e.g., Skilling 1975). The former cascade operates in the perpendicular wavenumber component (and involves a population of nearly perpendicular waves, $A_{\perp}$, and oblique waves, $A_{\swarrow}^{ \pm}$) and the latter one in the parallel component. The kinetic equations for these processes, easily adaptable to a Monte Carlo simulation, can be obtained using quantum-mechanical formalism (Melrose 1980). Both cascades lead to an effective damping of the resonant waves. The parallel cascade, however, also helps spreading the wave power to wavenumbers, where the resonant particles are unable to generate waves on their own. In addition, it provides waves propagating in the stable direction, so it may also lead to efficient stochastic acceleration of resonant particles. It is left for future simulations to address the effects of the wave-wave interactions to the flare scenario.

We have neglected particle drifts due to field curvature in our simulations, which is justified because of the long time scales (hours) associated with the curvature drift (Ramaty \& Mandzhavidze 1994). We have also neglected Coulomb losses and nuclear interactions during the propagation in our model. This is justified, since the trapping times we consider are relatively short $(\$ 10 \mathrm{~s})$ : a Coulomb-loss estimate (e.g., Hulot et al. 1989) gives 
a momentum-loss rate of $(\dot{p} / p)_{\mathrm{c}}=-3 \times 10^{-3} \mathrm{~s}^{-1}$ at $E=30 \mathrm{MeV}$ and $n_{0}=10^{11} \mathrm{~cm}^{-3}$.

In conclusion, the impulsive flare scenario in light of our simulations is the following: promptly $(t \lesssim 1 \mathrm{~s})$ after the start of the proton acceleration process near the loop top, the footpoints will brighten in $\gamma$-rays because the the turbulent trap will not be developed at that time. For a high level of ambient turbulence or large loop length, the footpoints may show rather different emission levels if the source is positioned somewhat off the loop top. After the first brightening, the turbulent particle trap develops and emission from the loop legs will stay at a level determined by the convective flux of particles. After one Alfvénic propagation time from the source, the closer loop leg will brighten in $\gamma$-rays once again, provided that the number of high-energy particles after the adiabatic losses in the trap is large enough, $p \mathrm{~d} N /(\mathrm{d} A \mathrm{~d} p) \gg(4 / \pi)\left(n_{0} V_{\mathrm{A}} / \Omega_{\mathrm{p}}\right) \sim 10^{13} n_{11}^{1 / 2} \mathrm{~cm}^{-2}$ with $n_{11}=n_{0} /\left(10^{11} \mathrm{~cm}^{-3}\right)$. The footpoint farther away from the source is unlikely to show the delayed peak in $\gamma$-ray emission in any case. We note that this feature is observationally testable, especially with the images of solar $\gamma$-ray emission anticipated from the HESSI mission. We also found that the small interplanetary-to-interacting proton ratios observed in impulsive solar flares do not necessarily imply closed field line topology, but can result also from the turbulent trapping on open magnetic field lines. In the next stage of the modeling, we intend to take better account of the resonance conditions and consider nonconstant background plasma parameters. The effects of wave-wave interactions will also be studied.

\section{Appendix A: Monte Carlo simulations of particle propagation through self-generated waves}

The present numerical code is modified from our previously published Monte Carlo code (Vainio et al. 2000). Similar transport codes, including also non-linear effects, have been developed previously (e.g., Ellison et al. 1996), but to our knowledge, ours is the first Monte Carlo code to employ self-generated waves.

When the Monte Carlo particles (numbered by $j$ ) are injected in the simulation, they are a given a weight $w_{j}$ that normalizes their injection rate to the physical value given by $Q$. The particles are treated in the guiding center approximation, which in the present case of constant magnetic field strength means that during the Monte Carlo time step, $\delta t$, we move the particles in the spatial coordinate (measured along the field lines) as $x_{j} \leftarrow x_{j}+v_{j} \mu_{j} \delta t$, where $v_{j}$ and $\mu_{j}$ are the particle speed and pitch-angle cosine of the $j$ th particle as measured in the fixed frame, where the background plasma is assumed to be stationary. In addition, the particles suffer scatterings from two Alfvén wave fields propagating parallel and anti-parallel to the magnetic field. In scatterings, performed after each Monte Carlo time step modeling pitch-angle diffusion, the particles are subsequently scattered (elastically) in the two wave frames, first Lorentz-transforming the particle velocity to the wave frame, then using

$$
\mu_{j \pm} \leftarrow \mu_{j \pm} \cos \vartheta_{j \pm}+\left(1-\mu_{j \pm}^{2}\right)^{1 / 2} \sin \vartheta_{j \pm} \cos \varphi_{j \pm}
$$

to compute the new wave-frame pitch-angle cosine $\mu_{j \pm}$, and finally transforming back to the fixed frame. Here $\vartheta_{j \pm}$ and $\varphi_{j \pm}$ are the scattering angles measured from and around the particle's velocity vector $\boldsymbol{v}_{ \pm}$prior to the scattering. As described in Vainio et al. (2000), $\vartheta_{j \pm}^{2}$ and $\varphi_{j \pm} \in[0,2 \pi)$ are random numbers picked up from exponential and uniform distributions, respectively, and ${ }^{1}$ $\left\langle\vartheta_{j \pm}^{2}\right\rangle=2 \nu_{j \pm} \delta t$, where $\nu_{j \pm} \propto U_{ \pm}\left(x_{j}, t\right)$ is the scattering frequency related to the wave-energy density by Eq. (1). Each Monte Carlo time step is chosen small enough to keep $\left\langle\vartheta_{j \pm}^{2}\right\rangle<0.2$ for all $j$.

We keep track of the wave energy densities on a spatial grid with $N=150$ elements numbered by $i=1, \ldots, N$, and with a spacing of $\Delta x=L / N$ and central coordinates $x=X_{i}$. The value of $U_{ \pm}(x)$ is taken to be constant inside each grid cell. Thus, $U_{ \pm}\left(x_{j}\right)=U_{ \pm}\left(X_{i_{j}}\right)$ and $i_{j}$ is the index of the grid cell that contains $x_{j}$, i.e., $x_{j} \in I_{i_{j}}$, where $I_{i}=$ $\left[X_{i}-\Delta x / 2, X_{i}+\Delta x / 2\right)$. During each Monte Carlo time step, a change of the wave energy density due to waveparticle interactions is computed from

$$
\begin{aligned}
& U_{ \pm}\left(X_{i}\right) \leftarrow \\
& \quad U_{ \pm}\left(X_{i}\right)+\frac{1}{A \Delta x} \sum_{x_{j} \in I_{i}} w_{j} \nu_{ \pm}\left(X_{j}\right) \delta t V_{\mathrm{A}} p_{j \pm} \mu_{j \pm}
\end{aligned}
$$

where $A$ is the cross-sectional area of the flux tube. This means that each Monte Carlo particle is taken to represent a large number of particles with the same parallel momentum, and the energy increment of the waves is computed from the mean value of the plasma-frame energy loss in wave-particle interactions of such particles. This method is much more stable computationally than an alternative method, where one computes the plasma-frame energy change in the scatterings of the Monte Carlo particles directly, and give this to the waves. After each Monte Carlo time step, we check that the wave-energy densities are above the minimum level given as a parameter.

In addition to the wave-particle interactions, the waves are convected on the grid by $U_{ \pm}\left(X_{i}\right) \leftarrow U_{ \pm}\left(X_{i \mp 1}\right)$ if $1 \leq i, i \mp 1 \leq N$ and $U_{+[-]}\left(X_{1[N]}\right)=U_{0}$, each time the simulation time $t$ has elapsed an amount of $\Delta x / V_{\mathrm{A}}$. At boundaries, the wave energy convected out of the grid is lost.

As an output, the simulation code saves the momentum, pitch-angle cosine and escape time of all particles leaving the simulation box. In addition, the wave-energy densities and the energetic particle pressure inside the simulation box is saved after every time the waves are moved on the grid, i.e., at $t \bmod \Delta x / V_{\mathrm{A}}=0$. To illustrate the typical development of a simulation, we have plotted in Fig. A.1 the wave-energy densities and particle pressures

1 Note that $\nu$ in Vainio et al. (2000) is defined as having half the value of $\nu_{ \pm}$in this work. 

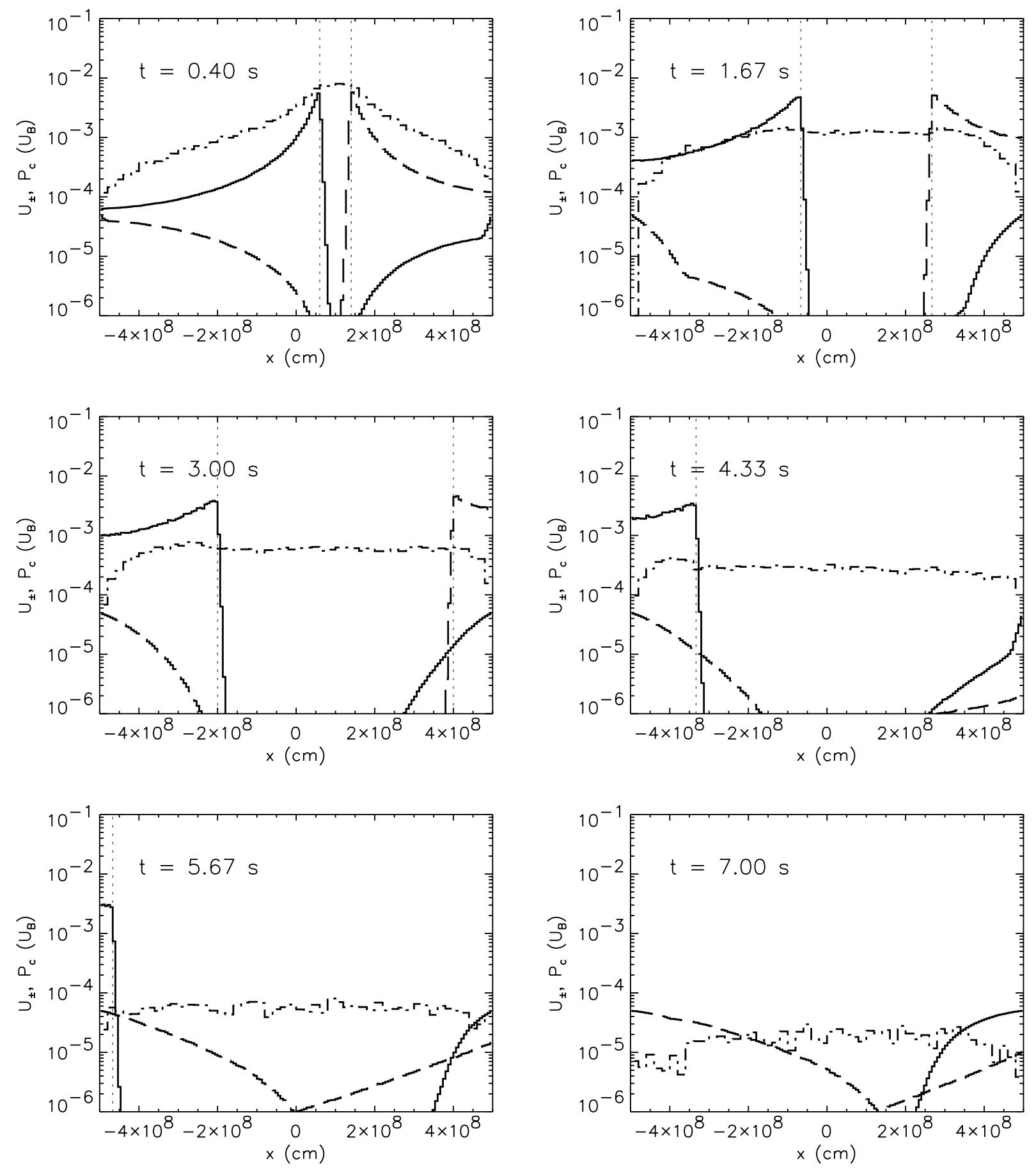

Fig. A.1. Snapshots from a simulation with an asymmetric position of the source (Case B), $x_{0}=0.1$ L. The solid (dashed) curve gives $U_{+(-)}$and the dashed-dotted curve the energetic-particle pressure $P_{\mathrm{c}}$ inside the loop as a function of position at indicated times after the start of the injection. The dotted lines give the positions $x=x_{0} \pm V_{\mathrm{A}} t$.

in a few frames for simulation in Case B with source position at $x=0.1 \mathrm{~L}$ (see Fig. 3 for the escaping particle flux).

As an outline of the future work, we note that a generalization to a spectrum of Alfvén waves would mean that the wave-energy density grid would contain another dimension (wavenumber), and that the resonance condition would have to be taken into account in deciding which particles contribute the the growth of the waves in the particular grid element. If one uses the full quasi-linear resonance condition with $\mu$-dependence, one has to also take this into account when modeling the scattering frequency, which has to be allowed a $\mu$-dependence. This, naturally, affects also the growth rate of the waves. The effects of non-constant magnetic field to the particle transport are easy to take into account (Vainio et al. 2000); 
for wave transport, one has to use an equation that employs the diverging field effects as well as the effects of a non-constant group speed.

\section{References}

Antonucci, E., \& Dodero, M. A. 1995, ApJ, 438, 480

Bell, A. R. 1978, MNRAS, 182, 147

Bespalov, P. A., Zaitsev, V. V., \& Stepanov, A. V. 1987, Solar Phys., 114, 127

Bespalov, P. A., Zaitsev, V. V., \& Stepanov, A. V. 1991, ApJ, 374,369

Cane, H. V., McGuire, R. E., \& von Rosenvinge, T. T. 1986, ApJ, 301, 448

Cliver, E. W. 1996, in High Energy Solar Physics, ed. R. Ramaty, N. Mandzhavidze, \& X.-M. Hua, AIP Conf. Proc., 374,45

Ellison, D. C., Baring, M. G., \& Jones, F. C. 1996, ApJ, 473, 1029

Hua, X.-M., \& Lingenfelter, R. E. 1987, Solar Phys., 107, 351

Hulot, E., Vilmer, N., \& Trottet, G. 1989, A\&A, 213, 383

Hulot, E., Vilmer, N., Chupp, E. L., Dennis, B. R., \& Kane, S. R. 1992, A\&A, 256, 273
Kocharov, L. G., \& Kovaltsov, G. A. 1986, in Proc. Int. School and Workshop on Plasma Astrophysics, ed. T. D. Guyenne (Noordwijk: ESA), SP-251, 101

Kocharov, L., Kovaltsov, G. A., \& Torsti, J. 1999, ApJ, 519, 422

Lee, M. A. 1983, JGR, 88, 6109

Mazur, V. A., \& Stepanov, A. V. 1984, A\&A, 139, 467

McClymont, A. N., \& Mikic, Z. 1994, ApJ, 422, 899

Melrose, D. B. 1980, Plasma Astrophysics, vol. I (Gordon and Breach: New York)

Ng, C. K., Reames, D. V., \& Tylka, A. J. 1999, Geophys. Res. Lett., 26, 2145

Ramaty, R., Mandzhavidze, N., Kozlovsky, B., \& Skibo, J. G. 1993, Adv. Space Res., 13(9), 275

Ramaty, R., \& Mandzhavidze, N. 1994, in High Energy Solar Phenomena, ed. J. M. Ryan, \& W. T. Vestrand, AIP Conf. Proc., 294, 26

Reames, D. V. 1999, Space Sci. Rev., 90, 413

Skilling, J. 1975, MNRAS, 173, 255

Vainio, R. 2000, ApJS, 131, 519

Vainio, R., Kocharov, L., \& Laitinen T. 2000, ApJ, 528, 1015

Wentzel, D. G. 1976, ApJ, 208, 595 